\title{
Cancer Drug Delivery in Three Dimensions For a Distributed Parameter Control Model Using Finite Elements*
}

\author{
Siddhartha P. Chakrabarty ${ }^{\dagger}$ and Floyd B. Hanson ${ }^{\dagger}$
}

\begin{abstract}
Optimal distributed parameter control and a Galerkin finite element method are used to develop procedures for an ideal model of optimal drug delivery to brain tumors. The mathematical model comprises of a system of three coupled reaction diffusion models, involving the density of tumor cells, normal tissue and also the drug concentration. An optimal control problem is formulated with the goal of minimizing the tumor cell density and reducing the side effects of the drug. A distributed parameter method based on a simple application of variational calculus is used on a pseudo-Hamiltonian, which is then used to obtain a coupled system of forward state equations and backward co-state equations. The Galerkin finite element method is used to realistically represent the brain structure. Finally, an ideal three dimensional test case is considered and partitioned into a set of brick finite elements in spherical coordinates, with tri-linear basis functions. Non-uniqueness of nodes in spherical coordinates is removed by combining like nodes, such as at the origin, at the poles and at the polar angle discontinuity. The Galerkin ODEs are solved by a combination of Crank-Nicolson and predictor-corrector methods.
\end{abstract}

\section{INTRODUCTION}

Various kinds of cancerous growth have been studied not only from the medical perspective, but also from the mathematical point of view. One such kind is the brain tumor, which like most cancerous cells originates from a single cell and proliferates into the neighboring normal cells. Understanding the mechanism behind the growth of tumors is necessary for designing an optimal treatment. Gliomas, a very deadly form of brain tumors, account for a majority of the cases, according to Murray [11] and Swanson [13]. Despite the advanced disgnostic procedures available, their benefits have been limited due to various impedimets like the existence of the blood brain barrier (BBB) [4]. The commonly used forms of drug delivery are drugs congugated with polymer and delivery by optimal distribution of drugs about the original tumor site. Wang et al. [14], [15] have worked extensively on drug delivery to tumors in three dimensions. The focus is on post-operative treatment of the resection arising from the removal of the bulk of the tumor.

The tumor-drug model here is from Chakrabarty and Hanson [2], [3], which was influenced by Gatenby et al. [5] and Mansuri [9]. Murray's fine text [10] is a good source of

\footnotetext{
${ }^{*}$ The work is supported by the National Science Foundation under Grant DMS-02-07081. The content of this material is that of the authors and does not necessarily reflect the views of the National Science Foundation.. The second author was also supported by the Provost's Award for Graduate Research awarded by the Graduate College at the University of Illinois at Chicago. Paper to appear in Proceedings of 45th Conference on Decision and Control, pp. 1-6, San Diego, CA, 13-15 December 2006.

$\dagger$ Department of Mathematics, Statistics, and Computer Science, University of Illinois at Chicago 851 S Morgan St.; m/c 249, Chicago, IL 606077045, USA, E-Mail: hanson@math.uic.edu and pratim@math.uic.edu
}

different types of growth mechanisms. The emphasis is on the development of analytical and computational techniques for future, larger inhomogeneous brain models with complex geometries.

The paper is organized in the following manner. In section II, we have the reaction diffusion mathematical model. In section III, the optimal distributed parameter control problem is formulated, a pseudo-Hamiltonian is defined and then calculus of variations [6] is applied to it to obtain a forward state equation and a backward co-state equation. In the next two sections we present the Galerkin finite element method and finite element test configuration using the spherical coordinates. Finally we present the computational results of numerical implementation of this finite element formulation.

\section{MAThematicAl Model}

In the distributed parameter control model of Chakrabarty and Hanson [2], [3], the tumor cell and normal cell density and the drug concentration at any position vector $\mathbf{x}$ and time $t \in\left[0, t_{f}\right]$, in the interior $\Omega$ of the domain, denoted by $n_{1}(\mathbf{x}, t), n_{2}(\mathbf{x}, t)$ and $c(\mathbf{x}, t)$ respectively, are taken as the state variables. Defining the global state vector as

$$
\mathbf{Y}(\mathbf{x}, t) \equiv\left[\begin{array}{lll}
n_{1}(\mathbf{x}, t) & n_{2}(\mathbf{x}, t) & c(\mathbf{x}, t)
\end{array}\right]^{\top},
$$

the governing nonlinear vector PDE is given by

$$
\mathbf{Y}_{t}(\mathbf{x}, t)=D \nabla_{x}^{2}[\mathbf{Y}]+(A+B)(\mathbf{Y}) \mathbf{Y}+\mathbf{U}
$$

where the nonlinearities are given by

$$
\begin{gathered}
A(\mathbf{Y})=a_{1}\left(1-Y_{1} / k_{1}\right) \mathbf{e}_{1} \mathbf{e}_{1}^{\top}+a_{2}\left(1-Y_{2} / k_{2}\right) \mathbf{e}_{2} \mathbf{e}_{2}^{\top}-a_{3} \mathbf{e}_{3} \mathbf{e}_{3}^{\top}, \\
B(\mathbf{Y})=-\left(\alpha_{1,2} Y_{2}+\kappa_{1,3} Y_{3}\right) \mathbf{e}_{1} \mathbf{e}_{1}^{\top}-\left(\alpha_{2,1} Y_{1}+\kappa_{2,3} Y_{3}\right) \mathbf{e}_{2} \mathbf{e}_{2}^{\top} \\
\mathbf{U}(\mathbf{x}, t)=U_{3}(\mathbf{x}, t) 1_{\mathbf{x} \in \mathcal{U} \mathbf{e}_{3}}
\end{gathered}
$$

where $\mathcal{U} \subset \Omega$ is the localized drug bolus input subset and $1_{\mathbf{x} \in \mathcal{U}}$ is the indicator function. Here, $D_{i}>0$ is the $i$ th component of the diagonal diffusion coefficient $D$ (could be inhomogeneous depending on the brain matter [13]), $A_{i, i}(\mathbf{Y}) Y_{i}$ is the $i$ th growth rate (logistic for $i=1: 2$ and exponentially decaying for $i=3$ for our test case), $\alpha_{i, j}$ are death rates due to competition, $\kappa_{i, j}$ are the death rates due to treatment and $u=U_{3}(\mathbf{x}, t)$ is the rate at which the drug is being delivered and will be the control variable in an optimal control system. Also, $\mathbf{e}_{i}$ is the $i$ th unit vector. The initial conditions and the no-flux boundary conditions on the boundary $\partial \Omega$ are, respectively,

$$
\begin{array}{r}
\mathbf{Y}(\mathbf{x}, 0)=\mathbf{Y}_{0}(\mathbf{x}), \\
-D\left(\widehat{\mathbf{N}} \cdot \nabla_{x}\right)[\mathbf{Y}](\mathbf{x}, t)=\mathbf{0} .
\end{array}
$$




\section{The Optimal Control Problem}

The quadratic objective functional for combined running and terminal costs is given by,

$$
\begin{aligned}
J[\mathbf{Y}, \mathbf{U}]= & \frac{1}{2} \int_{0}^{t_{f}} d t \int_{\Omega} d \mathbf{x}\left(\mathbf{Y}^{\top} R \mathbf{Y}+\left(\mathbf{U}-\mathbf{U}_{0}\right)^{\top} S\left(\mathbf{U}-\mathbf{U}_{0}\right)\right) \\
& +\frac{1}{2} \int_{\Omega} d \mathbf{x}\left(\mathbf{Y}^{\top} Q \mathbf{Y}\right)\left(\mathbf{x}, t_{f}\right),
\end{aligned}
$$

where coefficients are $R=r_{1} \mathbf{e}_{1} \mathbf{e}_{1}^{\top}$ for state costs, $S=$ $s_{3} \mathbf{e}_{3} \mathbf{e}_{3}^{\top}$ for control costs and $Q=q_{1} \mathbf{e}_{1} \mathbf{e}_{1}^{\top}+q_{3} \mathbf{e}_{3} \mathbf{e}_{3}^{\top}$ for final costs, while $\mathbf{U}_{0}=U_{0,3}(\mathbf{x}, t) 1_{\mathbf{x} \in \mathcal{U}} \mathbf{e}_{3}$ is some threshold rate. The goal is to minimize this functional with respect to the drug input rate relative to a minimal effectiveness rate $U_{0,3}$ and the terminal costs at $t_{f}$, i.e., $\min _{u}[J(u)]$ also reduces the effects of toxicity. Note that here $r_{1}>0$ is the tumor burden cost coefficient and $s_{3}>0$ is the drug delivery cost coefficient, while $q_{1}>0$ and $q_{3}>0$ are the corresponding final costs. In addition, no assumption is made about the control constraints like physical restriction on the amount of drugs that can be administered.

Using three Lagrange multiplier vectors, two of which are functions of space and time and one is independent of time, and letting $\mathbf{Z}=(\mathbf{Y}, \mathbf{U}, \boldsymbol{\xi}, \boldsymbol{\eta}, \boldsymbol{\chi})$ be an extended state vector, we define the pseudo-Hamiltonian as

$$
\begin{aligned}
\mathcal{H}(\mathbf{Z}) \equiv & \frac{1}{2} \int_{0}^{t_{f}} d t \int_{\Omega} d \mathbf{x}\left(\mathbf{Y}^{\top} R \mathbf{Y}+\left(\mathbf{U}-\mathbf{U}_{0}\right)^{\top} S\left(\mathbf{U}-\mathbf{U}_{0}\right)\right) \\
& +\frac{1}{2} \int_{\Omega} d \mathbf{x}\left(\mathbf{Y}^{\top} Q \mathbf{Y}\right)\left(\mathbf{x}, t_{f}\right) \\
& +\int_{0}^{t_{f}} d t \int_{\Omega} d \mathbf{x} \boldsymbol{\xi}^{\top}\left(\mathbf{Y}_{t}-D \nabla_{x}^{2}[\mathbf{Y}]-(A+B)(\mathbf{Y}) \mathbf{Y}-\mathbf{U}\right) \\
& +\int_{0}^{t_{f}} d t \int_{\partial \Omega} d \mathbf{\Gamma} \boldsymbol{\eta}^{\top}\left(-D\left(\widehat{\mathbf{N}} \cdot \nabla_{x}\right)[\mathbf{Y}]\right) \\
& +\int_{\Omega} d \mathbf{x}\left(\chi^{\top}\left(\mathbf{Y}-\mathbf{Y}_{0}\right)\right)(\mathbf{x}, 0) .
\end{aligned}
$$

The calculus of variations simplifies the analysis by using it to determine the critical point necessary condition for the first variation [6] of the pseudo-Hamiltonian $\mathcal{H}(\mathbf{Z})$. Let the perturbation $\delta \mathbf{Z}$ about the optimal trajectory $\mathbf{Z}^{*}$, be defined as $\delta \mathbf{Z}=\mathbf{Z}-\mathbf{Z}^{*}$. The pseudo-Hamiltonian is expanded as

$$
\mathcal{H}\left(\mathbf{Z}^{*}+\delta \mathbf{Z}\right)=\mathcal{H}\left(\mathbf{Z}^{*}\right)+\delta \mathcal{H}\left(\mathbf{Z}^{*}, \delta \mathbf{Z}\right)+O\left((\delta \mathbf{Z})^{2}\right) .
$$

The quadratic order terms of $\mathcal{H}$ are neglected. Also the functional dependence of the higher derivatives in time and state of the extended state perturbations must be eliminated on lower order terms by one or two integrations by parts, (using Green's formula [7]). Rearranging inner products and collecting terms, the extended state equations yields an intermediate form (see [1], [2], [3] for details). The resulting coefficients of independent variations are set to zero to obtain the state, control and co-state equations.

\section{A. State Equations}

The optimal state equation is recovered by setting the coefficient of $(\delta \boldsymbol{\xi})^{\top}$ to zero:

$$
\mathbf{Y}_{t}^{*}=D \nabla_{x}^{2}\left[\mathbf{Y}^{*}\right]+(A+B)\left(\mathbf{Y}^{*}\right) \mathbf{Y}^{*}+\mathbf{U}^{*}
$$

on $\Omega \times\left(0, t_{f}\right]$, with boundary conditions on $\partial \Omega \times\left[0, t_{f}\right]$ from the coefficient of $(\delta \boldsymbol{\eta})^{\top}$, i.e.,

$$
-D\left(\widehat{\mathbf{N}} \cdot \nabla_{x}\right)\left[\mathbf{Y}^{*}\right](\mathbf{x}, t)=\mathbf{0},
$$

for $(\mathbf{x}, t) \in \partial \Omega \times\left[0, t_{f}\right]$ and with initial conditions on the interior $\Omega$ from the coefficient of $(\delta \chi)^{\top}$, i.e.,

$$
\mathbf{Y}^{*}(\mathbf{x}, 0)=\mathbf{Y}_{0}(\mathbf{x})
$$

for $\mathbf{x} \in \Omega$. Due to the presence of the functions $A(\mathbf{Y}) \mathbf{Y}$ and $B(\mathbf{Y}) \mathbf{Y}$ the forward PDE (8) will be nonlinear.

\section{B. Regular Optimal Control}

Since the control has been defined in (3) with only one component, only the coefficient of $\delta U_{3}$ is set to zero giving the corresponding drug regular control

$$
U_{3}^{*}(\mathbf{x}, t)=U_{0,3}(\mathbf{x}, t)+\xi_{3}^{*}(\mathbf{x}, t) 1_{\mathbf{x} \in \mathcal{U}} / s_{3},
$$

on $\Omega \times\left[0, t_{f}\right]$, since $\delta U_{3} \equiv 0$ for $\mathbf{x} \notin \mathcal{U}$ and provided $s_{3} \neq 0$. Note that this control law only requires solving for the $3 \mathrm{rd}$ component of $\boldsymbol{\xi}^{*}(\mathbf{x}, t)$, since $\delta U_{1} \equiv 0$ and $\delta U_{2} \equiv 0$.

\section{Co-State Equations}

Setting the functional coefficient of $(\delta \mathbf{Y})^{\top}$ to zero yields the primary co-state backward PDE:

$$
\begin{aligned}
\mathbf{0}= & \boldsymbol{\xi}_{t}^{*}+\nabla_{x}^{2}\left[D \boldsymbol{\xi}^{*}\right]+(A+B)\left(\mathbf{Y}^{*}\right) \boldsymbol{\xi}^{*} \\
& +\nabla_{Y}[A+B]\left(\mathbf{Y}^{*}\right):\left(\boldsymbol{\xi}^{*}\left(\mathbf{Y}^{*}\right)^{\top}\right)-R \mathbf{Y}^{*},
\end{aligned}
$$

for $(\mathbf{x}, t) \in \Omega \times\left[0, t_{f}\right)$. This PDE (12) is unidirectionally coupled to the state PDE (8). The boundary condition is from setting the coefficient of $\delta \mathbf{Y}(\mathbf{x}, t)$ for $\mathbf{x}$ on $\boldsymbol{\Gamma}=\partial \Omega$ to zero, so

$$
\left(\widehat{\mathbf{N}} \cdot \nabla_{x}\right)\left[D \boldsymbol{\xi}^{*}\right](\mathbf{x}, t)=\mathbf{0}, \quad(\mathbf{x}, t) \in \partial \Omega \times\left[0, t_{f}\right)
$$

and the final condition for this backward PDE follows from forcing the coefficient of $\delta \mathbf{Y}\left(\mathbf{x}, t_{f}\right)$ to be zero on $\Omega$,

$$
\boldsymbol{\xi}^{*}\left(\mathbf{x}, t_{f}\right)=-Q \mathbf{Y}^{*}\left(\mathbf{x}, t_{f}\right) .
$$

The two other co-state vectors should not be needed, but satisfy rather simple equations [2], [3]. However, in general, there are 6 vector PDEs in 3 dimensional space, leading to a very high computational complexity when converted to numerical methods.

\section{Galerkin Finite Element Method}

In [2], [3] the Crank-Nicolson implicit method combined with predictor-corrector methods are used to study the problem numerically. However, using finite difference methods like Crank-Nicolson implicit method alone and alternating directions implicit method have serious drawbacks. Finite difference techniques are more likely to have higher computational requirements, i.e, more likely suffering from the curse of dimensionality. Finite element methods require a relatively smaller number of nodes as compared to the finite difference methods while maintaining the same level of accuracy. Also, the finite element method can better handle irregular structure, such as a brain tumor or ventricles. Hanson [8] has worked extensively in this area and has made a comparative study of different numerical methods for stochastic dynamic programming. For the problem under consideration, the Galerkin finite element method is used so as to reduce the number of state nodes. The following steps can be used to get an numerical approximation.

1) The first forward step $(\delta=1)$ of forward-backward iteration is to guess an initial control $U_{3}^{*}(\mathbf{x}, t) \simeq$ 
$U_{3}^{(1)}(\mathbf{x}, t)$ for forward state equations and use the finite element method to solve for the state $\mathbf{Y}^{*}(\mathbf{x}, t) \simeq$ $\mathbf{Y}^{(1)}(\mathbf{x}, t)$ for $t>0$. Initially, $\mathbf{Y}^{*}(\mathbf{x}, 0)=\mathbf{Y}_{0}(\mathbf{x})$. Let the Galerkin approximation for the optimal state and control be

$$
\begin{aligned}
& \mathbf{Y}^{*}(\mathbf{x}, t) \simeq \widehat{\mathbf{Y}}^{(\delta)}(\mathbf{x}, t) \equiv \sum_{\hat{k}=1}^{\widehat{M}} \widehat{\mathbf{Y}}_{\hat{k}}^{(\delta)}(t) \cdot \phi_{\hat{k}}(\mathbf{x}) \\
& \mathbf{U}^{*}(\mathbf{x}, t) \simeq \widehat{\mathbf{U}}^{(\delta)}(\mathbf{x}, t) \equiv 1_{\mathbf{x} \in \mathcal{U}} \sum_{\hat{k}=1}^{\widehat{M}} \widehat{\mathbf{U}}_{\hat{k}}^{(\delta)}(t) \cdot \phi_{\hat{k}}(\mathbf{x}),
\end{aligned}
$$

where, $\left[\phi_{i}(\mathbf{x})\right]_{\widehat{M} \times 1}$, is a set of $\widehat{M}$ linearly independent continuous basis functions, with the interpolation condition $\phi_{\hat{k}}\left(\mathbf{x}_{\hat{j}}\right)=\delta_{\hat{j}, \hat{k}}$, at the element node $\mathbf{x}_{\hat{j}}$, implying that $\mathbf{Y}^{*}\left(\mathbf{x}_{\hat{j}}, t\right)=\widehat{\mathbf{Y}}_{\hat{j}}^{(\delta)}(t)$ for $\hat{j}=1: \widehat{M}$ element nodes.

2) Before applying the Galerkin approximations, the integral form of the state equation (8) with respect to a basis test function $\phi_{\hat{j}}(\mathbf{x})$ is needed,

$$
\begin{aligned}
\mathbf{0}= & \int_{\Omega} d \mathbf{x} \phi_{\hat{j}}(\mathbf{x})\left(\mathbf{Y}_{t}^{*}-D \nabla_{x}^{2}\left[\mathbf{Y}^{*}\right]-(A+B)\left(\mathbf{Y}^{*}\right) \mathbf{Y}^{*}-\mathbf{U}^{*}\right) \\
= & \int_{\Omega} d \mathbf{x}\left(\phi_{\hat{j}} \mathbf{Y}_{t}^{*}+D \nabla_{x}^{\top}\left[\phi_{\hat{j}}\right] \nabla_{x}\left[\mathbf{Y}^{*}\right]\right. \\
& \left.-\phi_{\hat{j}}\left((A+B)\left(\mathbf{Y}^{*}\right) \mathbf{Y}^{*}+\mathbf{U}^{*}\right)\right)
\end{aligned}
$$

for $\hat{j}=1: \widehat{M}$, where the 2 nd order derivatives have been reduced to 1 st order derivatives by integration by parts (Green's formula [7]) and the exact no-flux boundary condition has been used.

3) Now, the Galerkin approximations (15-16) are applied

$$
\begin{aligned}
\mathbf{0} \simeq & \sum_{\hat{k}=1}^{\widehat{M}} \int_{\Omega} d \mathbf{x}\left(\widehat{\mathbf{Y}}_{\hat{k}}^{\prime} \phi_{\hat{j}} \phi_{\hat{k}}+D \widehat{\mathbf{Y}}_{\hat{k}}\left(\nabla_{x}^{\top}\left[\phi_{\hat{j}}\right] \nabla_{x}\left[\phi_{\hat{k}}\right]\right)\right. \\
& \left.-\left((A+B)(\widehat{\mathbf{Y}}) \widehat{\mathbf{Y}}_{\hat{k}}+\widehat{\mathbf{U}}_{\hat{k}}\right) \phi_{\hat{j}} \phi_{\hat{k}}\right),
\end{aligned}
$$

for $\hat{j}=1: \widehat{M}$. Futher reduction to finite element integrals for $\hat{j}, \hat{k}, \hat{l}=1: \widehat{M}$ is accomplished by letting

$$
\widehat{\mathcal{M}}_{\hat{j}, \hat{k}} \equiv \int_{\Omega} d \mathbf{x} \phi_{\hat{j}}(\mathbf{x}) \phi_{\hat{k}}(\mathbf{x})
$$

be an element mass integral

$$
\widehat{\mathcal{K}}_{\hat{j}, \hat{k}} \equiv \int_{\Omega} d \mathbf{x} \nabla_{x}^{\top}\left[\phi_{\hat{j}}\right] \nabla_{x}\left[\phi_{\hat{k}}\right]
$$

be an element stiffness integral and

$$
\widehat{\mathcal{T}}_{\hat{j}, \hat{k}, \hat{l}} \equiv \int_{\Omega} d \mathbf{x} \phi_{\hat{j}}(\mathbf{x}) \phi_{\hat{k}}(\mathbf{x}) \phi_{\hat{l}}(\mathbf{x})
$$

be a triple basis element integral arising from the purely bilinear terms in $A(\mathbf{Y}) \mathbf{Y}$ and $B(\mathbf{Y}) \mathbf{Y}$. Thus, the Galerkin equation becomes,

$$
\begin{aligned}
\mathbf{0}= & \sum_{\hat{k}=1}^{\widehat{M}}\left(\widehat { \mathcal { M } } _ { \hat { j } , \hat { k } } \left(\widehat{\mathbf{Y}}_{\hat{k}}^{\prime}(t)-\left(a_{1} \mathbf{e}_{1} \mathbf{e}_{1}^{\top}+a_{2} \mathbf{e}_{2} \mathbf{e}_{2}^{\top}\right.\right.\right. \\
& \left.\left.-a_{3} \mathbf{e}_{3} \mathbf{e}_{3}^{\top}\right) \widehat{\mathbf{Y}}_{\hat{k}}(t)-\widehat{\mathbf{U}}_{\hat{k}}(t)\right)+D \widehat{\mathcal{K}}_{\hat{j}, \hat{k}} \widehat{\mathbf{Y}}_{\hat{k}}(t) \\
& +\sum_{\hat{M}}^{\widehat{\mathcal{T}}} \widehat{\mathcal{J}}_{\hat{j}, \hat{k}, \hat{l}}\left(\frac{a_{1}}{k_{1}} \widehat{Y}_{1, \hat{k}}(t) \widehat{Y}_{1, \hat{l}}(t) \mathbf{e}_{1}+\frac{a_{2}}{k_{2}} \widehat{Y}_{2, \hat{k}}(t) \widehat{Y}_{2, \hat{l}}(t) \mathbf{e}_{2}\right. \\
& +\left(\alpha_{1,2} \widehat{Y}_{2, \hat{l}}(t)+\kappa_{1,3} \widehat{Y}_{3, \hat{l}}(t)\right) \widehat{Y}_{1, \hat{k}}(t) \mathbf{e}_{1} \\
& \left.\left.+\left(\alpha_{2,1} \widehat{Y}_{1, \hat{l}}(t)+\kappa_{2,3} \widehat{Y}_{3, \hat{l}}(t)\right) \widehat{Y}_{2, \hat{k}}(t) \mathbf{e}_{2}\right)\right)
\end{aligned}
$$

for $\hat{j}=1: \widehat{M}$ (dropping the $(\delta)$ superscript here for brevity). This Galerkin ODE can be solved by approximating the Galerkin basis integral coefficients $\left(\widehat{\mathcal{M}}_{\hat{j}, \hat{k}}, \widehat{\mathcal{K}}_{\hat{j}, \hat{k}}, \widehat{\mathcal{T}}_{\hat{j}, \hat{k}, \hat{l}}\right)$ using symbolic methods and twopoint Gaussian quadrature in case of singularities, and then the ODE system is solved by the combined Crank-Nicolson and predictor-corrector methods. The coefficients can be computed for all double shots for fixed finite elements off-line since they will be fixed. These coefficients can be calculated on an element-byelement decomposition and element results can later be reassembled to form the global solution [12].

4) In the second, backward shot of the double shot algorithm [2], the final condition (14),

$$
\boldsymbol{\xi}^{(\delta)}\left(\mathbf{x}, t_{f}\right) \simeq-Q \widehat{\mathbf{Y}}^{(\delta)}\left(\mathbf{x}, t_{f}\right)=-Q \sum_{\hat{k}=1}^{\widehat{M}} \widehat{\mathbf{Y}}_{\hat{k}}^{(\delta)}\left(t_{f}\right) \phi_{\hat{k}}(\mathbf{x})
$$

for $\delta=1: L$ double shots, is used to start the backward co-state solution. Similar to the state equation, a Galerkin approximation for the co-state equation using the same basis is given by,

$$
\boldsymbol{\xi}^{*}(\mathbf{x}, t) \simeq \widehat{\boldsymbol{\xi}}^{(\delta)}(\mathbf{x}, t) \equiv \sum_{\hat{k}=1}^{\widehat{M}} \widehat{\boldsymbol{\xi}}_{\hat{k}}^{(\delta)}(t) \cdot \phi_{\hat{k}}(\mathbf{x})
$$

for $t<t_{f}$. Similar to the derivation of the state Galerkin finite element ODEs (20), the co-state Galerkin ODEs are

$$
\begin{aligned}
\mathbf{0}= & \sum_{\hat{k}=1}^{\widehat{M}}\left(\widehat { \mathcal { M } } _ { \hat { j } , \hat { k } } \left(\widehat{\boldsymbol{\xi}}_{\hat{k}}^{\prime}(t)+a_{1} \widehat{\xi}_{1, \hat{k}} \mathbf{e}_{1}+a_{2} \widehat{\xi}_{2, \hat{k}} \mathbf{e}_{2}\right.\right. \\
& \left.-a_{3} \widehat{\xi}_{3, \hat{k}} \mathbf{e}_{3}-R \widehat{\mathbf{Y}}_{\hat{k}}(t)\right)-D \widehat{\mathcal{K}}_{\hat{j}, \hat{k}} \widehat{\boldsymbol{\xi}}_{\hat{k}}(t) \\
& -\sum_{\hat{l}=1}^{\widehat{M}} \widehat{\mathcal{T}}_{\hat{j}, \hat{k}, \hat{l}}\left(\frac{2 a_{1}}{k_{1}} \widehat{Y}_{1, \hat{l}}(t) \widehat{\xi}_{1, \hat{k}}(t) \mathbf{e}_{1}\right. \\
& +\frac{2 a_{2}}{k_{2}} \widehat{Y}_{2, \hat{l}}(t) \widehat{\xi}_{2, \hat{k}}(t) \mathbf{e}_{2} \\
& +\alpha_{1,2}\left(\widehat{Y}_{2, \hat{l}}(t) \mathbf{e}_{1}+\widehat{Y}_{1, \hat{l}}(t) \mathbf{e}_{2}\right) \widehat{\xi}_{1, \hat{k}}(t) \\
& +\kappa_{1,3}\left(\widehat{Y}_{3, \hat{l}}(t) \mathbf{e}_{1}+\widehat{Y}_{1, \hat{l}}(t) \mathbf{e}_{3}\right) \widehat{\xi}_{1, \hat{k}}(t) \\
& +\alpha_{2,1}\left(\widehat{Y}_{2, \hat{l}}(t) \mathbf{e}_{1}+\widehat{Y}_{1, \hat{l}}(t) \mathbf{e}_{2}\right) \widehat{\xi}_{1, \hat{k}}(t) \\
& \left.\left.+\kappa_{2,3}\left(\widehat{Y}_{3, \hat{l}}(t) \mathbf{e}_{2}+\widehat{Y}_{2, \hat{l}}(t) \mathbf{e}_{3}\right) \widehat{\xi}_{2, \hat{k}}(t)\right)\right),
\end{aligned}
$$


for $\hat{j}=1: \widehat{M}$. This Galerkin ODE (22) may be computed by the appropriate numerical methods using the same Galerkin integral basis coefficients.

5) For each completed double shot for $\delta=1: L$, the costate approximation $\widehat{\boldsymbol{\xi}}^{(\delta)}(\mathbf{x}, t)(21)$ is for the regular optimal control (11) $\widehat{U}_{3}$ update,

$$
\widehat{U}_{3}^{(\delta+1)}(\mathbf{x}, t)=U_{0,3}(\mathbf{x}, t)+\widehat{\xi}_{3}^{(\delta)}(\mathbf{x}, t) / s_{3}, \quad \mathbf{x} \in \mathcal{U} .
$$

6) This process is repeated for $\delta=2: L$ double shot iterations until a convergence criterion for a sufficient $L$ is reached, e.g., the relative criterion for the control and the state

$$
\begin{aligned}
& \left\|U_{3}^{(\delta)}(\mathbf{x}, t)-U_{3}^{(\delta-1)}(\mathbf{x}, t)\right\|<\operatorname{tol}_{u}\left\|U_{3}^{(\delta-1)}(\mathbf{x}, t)\right\|, \\
& \left\|\mathbf{Y}^{(\delta)}(\mathbf{x}, t)-\mathbf{Y}^{(\delta-1)}(\mathbf{x}, t)\right\|<\operatorname{tol}_{y}\left\|\mathbf{Y}^{(\delta-1)}(\mathbf{x}, t)\right\|,
\end{aligned}
$$

respectively, for $\delta=2: L$ until satisfied, provided $\left\|U_{3}^{(\delta-1)}(\mathbf{x}, t)\right\| \neq 0$ and $\left\|\mathbf{Y}^{(\delta-1)}(\mathbf{x}, t)\right\| \neq 0$, where $\operatorname{tol}_{u}>0$ and $\operatorname{tol}_{y}>0$ are relative prescribed tolerances.

\section{Spherical Finite Element Test Configuration}

Consider a three dimensional test configuration that is a sphere of radius $R_{r}$. Transforming the spherical coordinates in space as usual,

$$
(x, y, z)=r(\cos (\theta) \sin (\psi), \sin (\theta) \sin (\psi), \cos (\psi))
$$

where $r, \theta, \psi$ are the radius, polar angle and azimuthal angle, respectively. Also, $0 \leq r \leq R_{r}, 0 \leq \theta \leq 2 \pi$ and $0 \leq \psi \leq \pi$. The brick element grid in spherical coordinates is constructed of $M_{r}$ radial sectors of width $\Delta r=R_{r} / M_{r}, M_{\theta}$ polar sectors of width $\Delta \theta=2 \pi / M_{\theta}$ and $M_{\psi}$ azimuthal sectors of width $\Delta \psi=\pi / M_{\psi}$. The nodal values are given by $\left(\theta_{i_{e}}, \psi_{j_{e}}, r_{k_{e}}\right)=\left(\left(i_{e}-1\right) \Delta \theta,\left(j_{e}-1\right) \Delta \psi,\left(k_{e}-1\right) \Delta r\right)$ for $i_{e}=$ $1: M_{\theta}+1, j_{e}=1: M_{\psi}+1$ and $k_{e}=1: M_{r}+1$. The elements are numbered in $(\theta, \psi, r)$ linear priority order like the nodal values, $e_{i_{e}, j_{e}, k_{e}}=i_{e}+\left(j_{e}-1\right) \cdot M_{\theta}+\left(k_{e}-1\right) \cdot M_{\theta} \cdot M_{\psi}$,for $i_{e}=1: M_{\theta}, j_{e}=1: M_{\psi}$ and $k_{e}=1: M_{r}$. Within element $e_{i_{e}, j_{e}, k_{e}}$, the element primary node with local node number $i=1$ has the same global node number $\bar{k}_{e}=\left\{i_{e}, j_{e}, k_{e}\right\}$ as the element, i.e., $n_{\bar{k}_{e}, 1}=e_{i_{e}, j_{e}, k_{e}}$ for $i_{e}=1: M_{\psi}$, $j_{e}=1: M_{\theta}, k_{e}=1: M_{r}$. The element local node numbering is $i=1: 8$ as shown in the Figure 1 . The formulation is given in

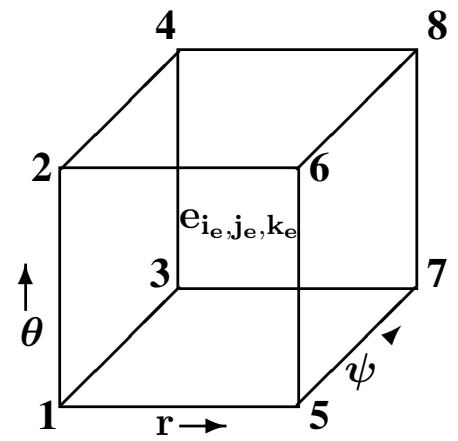

Fig. 1. Local node numbering with $i=1: 8$ for general element $e_{i_{e}, j_{e}, k_{e}}$. some detail here, since hardy any useful details on spherical elements are given in the literature to our knowledge.
For simplicity, trilinear basis functions are used for all brick elements in spherical coordinates. The trilinear bases are constructed from the more basic one-dimensional linear bases with only two nodes labeled 1 and 2:

$$
\begin{aligned}
\phi_{1 r}^{\left(k_{e}\right)}(r)=\left(\frac{r_{k_{e}+1}-r}{\Delta r}\right), & \phi_{2 r}^{\left(k_{e}\right)}(r)=\left(\frac{r-r_{k_{e}}}{\Delta r}\right) ; \\
\phi_{1 \theta}^{\left(i_{e}\right)}(\theta)=\left(\frac{\theta_{i_{e}+1}-\theta}{\Delta \theta}\right), & \phi_{2 \theta}^{\left(i_{e}\right)}(\theta)=\left(\frac{\theta-\theta_{i_{e}}}{\Delta \theta}\right) ; \\
\phi_{1 \psi}^{\left(j_{e}\right)}(\psi)=\left(\frac{\psi_{j_{e}+1}-\psi}{\Delta \psi}\right), & \phi_{2 \psi}^{\left(j_{e}\right)}(\psi)=\left(\frac{\psi-\psi_{j_{e}}}{\Delta \psi}\right) ;
\end{aligned}
$$

For the general element $e_{i_{e}, j_{e}, k_{e}}$ on $\left[\theta_{i_{e}}, \theta_{i_{e}+1}\right] \times\left[\psi_{j_{e}}, \psi_{j_{e}+1}\right] \times$ $\left[r_{k_{e}}, r_{k_{e}+1}\right]$, for $i_{e}=1: M_{\theta}, j_{e}=1: M_{\psi}$ and $k_{e}=2: M_{r}$ in the $i=1: 8$ element node numbering,

$$
\begin{aligned}
\phi_{1}^{\left(i_{e}, j_{e}, k_{e}\right)}(r, \theta, \psi) & =\phi_{1 r}^{\left(k_{e}\right)}(r) \cdot \phi_{1 \theta}^{\left(i_{e}\right)}(\theta) \cdot \phi_{1 \psi}^{\left(j_{e}\right)}(\psi) ; \\
\cdot \phi_{2}^{\left(i_{e}, j_{e}, k_{e}\right)}(r, \theta, \psi) & =\phi_{1 r}^{\left(k_{e}\right)}(r) \cdot \phi_{2 \theta}^{\left(i_{e}\right)}(\theta) \cdot \phi_{1 \psi}^{\left(j_{e}\right)}(\psi) ; \\
\phi_{3}^{\left(i_{e}, j_{e}, k_{e}\right)}(r, \theta, \psi) & =\phi_{1 r}^{\left(k_{e}\right)}(r) \cdot \phi_{1 \theta}^{\left(i_{e}\right)}(\theta) \cdot \phi_{2 \psi}^{\left(j_{e}\right)}(\psi) \\
\phi_{4}^{\left(i_{e}, j_{e}, k_{e}\right)}(r, \theta, \psi) & =\phi_{1 r}^{\left(k_{e}\right)}(r) \cdot \phi_{2 \theta}^{\left(i_{e}\right)}(\theta) \cdot \phi_{2 \psi}^{\left(j_{e}\right)}(\psi) ; \\
\phi_{5}^{\left(i_{e}, j_{e}, k_{e}\right)}(r, \theta, \psi) & =\phi_{2 r}^{\left(k_{e}\right)}(r) \cdot \phi_{1 \theta}^{\left(i_{e}\right)}(\theta) \cdot \phi_{1 \psi}^{\left(j_{e}\right)}(\psi) ; \\
\phi_{6}^{\left(i_{e}, j_{e}, k_{e}\right)}(r, \theta, \psi) & =\phi_{2 r}^{\left(k_{e}\right)}(r) \cdot \phi_{2 \theta}^{\left(i_{e}\right)}(\theta) \cdot \phi_{1 \psi}^{\left(j_{e}\right)}(\psi) ; \\
\phi_{7}^{\left(i_{e}, j_{e}, k_{e}\right)}(r, \theta, \psi) & =\phi_{2 r}^{\left(k_{e}\right)}(r) \cdot \phi_{1 \theta}^{\left(i_{e}\right)}(\theta) \cdot \phi_{2 \psi}^{\left(j_{e}\right)}(\psi) ; \\
\phi_{8}^{\left(i_{e}, j_{e}, k_{e}\right)}(r, \theta, \psi) & =\phi_{2 r}^{\left(k_{e}\right)}(r) \cdot \phi_{2 \theta}^{\left(i_{e}\right)}(\theta) \cdot \phi_{2 \psi}^{\left(j_{e}\right)}(\psi) .
\end{aligned}
$$

The mapping (24) from spherical to cartesian coordinates is not unique since the mapping is a many-to-one. The nonunique nodes arise at the origin for $r=r_{1}=0$ and any $(\theta, \psi)$ or at the poles for $\psi=0, \pi$ and any $(r, \theta)$ or at the periodic boundary condition when $\theta=2 \pi$ for any $(r, \psi)$ The non-uniqueness or resulting over-determinism can simply be removed by adding together the appropriate bases in (28)(31) corresponding to the same non-unique nodes, using the identities for the one-dimensional bases and their derivatives,

$$
\phi_{1 \rho}^{\left(\ell_{e}\right)}(\rho)+\phi_{2 \rho}^{\left(\ell_{e}\right)}(\rho)=1, \quad \phi_{1 \rho}^{\left(\ell_{e}\right){ }^{\prime}}(\rho)+\phi_{2 \rho}^{\left(\ell_{e}\right){ }^{\prime}}(\rho)=0
$$

for $\rho=r, \theta$ or $\psi$ and $\ell_{e}=k_{e}, i_{e}$ or $j_{e}$. While it may appear awkward to have to make this adjustment, the disadvantage is out-weighed by the ease of deforming a sphere into a brain geometry than deforming a brick or rectangular solid into a brain geometry. Another advantage of spherical coordinates is the ease of imposing the no-flux boundary condition $(B C)$ at $r=R_{r}$, since on the element $e_{i_{e}, j_{e}, k_{e}}$ the $i_{s}$ th state solution for $i_{s}=1: 3$ is expressed as a preliminary Galerkin approximation,

$Y_{i_{s}}^{\left(i_{e}, j_{e}, k_{e}\right)}(r, \theta, \psi, t) \simeq \sum_{j=1}^{8} \widetilde{Y}_{i_{s}, j}^{\left(i_{e}, j_{e}, k_{e}\right)}(t) \phi_{j}^{\left(i_{e}, j_{e}, k_{e}\right)}(r, \theta, \psi)$,

so the normal gradient, at boundary element $k_{e}=M_{r}$ with $r_{M_{r}+1}=R_{r}$ for local nodes $j=5: 8$, yields $Y_{i_{s}, r}^{\left(i_{e}, j_{e}, M_{r}\right)}\left(R_{r}, \theta, \psi, t\right)$ so that

$$
\widetilde{Y}_{i_{s}, j}^{\left(i_{e}, j_{e}, M_{r}\right)}(t)=\tilde{Y}_{i_{s}, j-4}^{\left(i_{e}, j_{e}, M_{r}\right)}(t)
$$

for $j=5: 8$ and arbitrary $(\theta, \psi)$ if $Y_{i_{s}, r}^{\left(i_{e}, j_{e}, M_{r}\right)}\left(R_{r}, \theta, \psi, t\right)=$ 0 with no-flux.This version of the no-flux condition is much better and simpler to use than that in [3], even when dealing with a deformed sphere in the form of a brain case.

The element matrices for node numbers $i, j, k=1: 8$ are 


$$
\begin{aligned}
\mathcal{M}_{i, j}^{\left(i_{e}, j_{e}, k_{e}\right)}= & \int_{\theta_{i_{e}}}^{\theta_{i_{e}+1}} d \theta \int_{\psi_{i_{e}}}^{\psi_{j_{e}+1}} d \psi \int_{r_{k_{e}}}^{r_{k_{e}+1}} d r r^{2} \sin (\psi) \\
& \left(\phi_{i}^{\left(i_{e}, j_{e}, k_{e}\right)} \phi_{j}^{\left(i_{e}, j_{e}, k_{e}\right)}\right)(r, \theta, \psi), \\
\mathcal{K}_{i, j}^{\left(i_{e}, j_{e}, k_{e}\right)}= & \int_{\theta_{i_{e}}}^{\theta_{i_{e}+1}} d \theta \int_{\psi_{i_{e}}}^{\psi_{j_{e}+1}} d \psi \int_{r_{k_{e}}}^{r_{k_{e}+1}} d r r^{2} \sin (\psi) \\
& \left(\phi_{i, r}^{\left(i_{e}, j_{e}, k_{e}\right)} \phi_{j, r}^{\left(i_{e}, j_{e}, k_{e}\right)}+\frac{1}{r^{2}} \phi_{i, \psi}^{\left(i_{e}, j_{e}, k_{e}\right)} \phi_{j, \psi}^{\left(i_{e}, j_{e}, k_{e}\right)}\right. \\
& \left.+\frac{1}{r^{2} \sin ^{2}(\psi)} \phi_{i, \theta}^{\left(i_{e}, j_{e}, k_{e}\right)} \phi_{j, \theta}^{\left(i_{e}, j_{e}, k_{e}\right)}\right)(r, \theta, \psi), \\
\mathcal{T}_{i, j, k}^{\left(i_{e}, j_{e}, k_{e}\right)}= & \int_{\theta_{i_{e}}}^{\theta_{i_{e}+1}} d \theta \int_{\psi_{i_{e}}}^{\psi_{j_{e}+1}} d \psi \int_{r_{k_{e}}}^{r_{k_{e}+1}} d r r^{2} \sin (\psi) \\
& \left(\phi_{i}^{\left(i_{e}, j_{e}, k_{e}\right)} \phi_{j}^{\left(i_{e}, j_{e}, k_{e}\right)} \phi_{k}^{\left(i_{e}, j_{e}, k_{e}\right)}\right)(r, \theta, \psi),
\end{aligned}
$$

dropping the matrix “へ” notation, where $\phi_{i, \rho}^{\left(i_{e}, j_{e}, k_{e}\right)}(\rho)$ denotes the partial derivative of $\phi_{i}^{\left(i_{e}, j_{e}, k_{e}\right)}(\rho)$ with respect to generic spherical coordinate $\rho$. In the stiffness matrix $\mathcal{K}$, the mapping singularities of the gradient lead to reciprocal factors in $r$ and $\sin (\psi)$, but the $r$ factors are simply cancelled by the Jacobian $r^{2} \sin (\psi)$ and leave uncanceled $\sin (\psi)$-denominators in the $\theta$-derivative term. However, these $\sin (\psi)$-denominators are completely eliminated in later analysis upon eliminating non-unique nodes by combining terms and associated ODEs.

\section{Computational Results}

The double shot iteration algorithm using the finite element method outlined in Section IV is implemented on three-dimensional space with the three states and the drug input control. The implementation is more similar to the two-dimensional problem treated in [3], except that the complexity of the mapping from spherical to cartesian is much greater than the polar mapping due to the degeneracy at the poles. Once the non-unique degeneracies of states and costates due to aliases and boundary conditions are eliminated so that there are only $\widehat{M}=\left(M_{r}-1\right)\left(M_{\theta}\left(M_{\psi}-1\right)+2\right)+1$ linearly independent Galerkin coefficients, $\hat{\mathbf{Y}}_{\hat{k}}(t)$ in ODE (20), $\widehat{\boldsymbol{\xi}}_{\hat{k}}(t)$ in ODE (22) and control $\widehat{\mathbf{U}}_{\hat{k}}(t)$. This nonuniqueness elimination keeps the system of ODEs from being over-determined, preserving the symmetry of the mass and other coefficient arrays, and eliminating the $1 / \sin (\psi)$ singularity in the stiffness integrals. For more details, see Chakrabarty's thesis [1].

The general method uses a combination of Crank-Nicolson and predictor-corrector methods developed in [8] for solving high dimensional stochastic control problems on supercomputers. The general method can handle both implicit and nonlinear terms. For simplicity, the forward ODE (20) for the $i_{s}$ th-state $\widehat{Y}_{i_{s}, \hat{j}}$ at node $\hat{j}$, with the degeneracies removed, is written symbolically, for $i_{s}=1: 3$ and nodes $\hat{j}=1: \widehat{M}$,

$$
\begin{aligned}
& \sum_{\hat{k}=1}^{\widehat{M}} \mathcal{M}_{\hat{j}, \hat{k}} \widehat{Y}_{i_{s}, \hat{k}}^{\prime}(t)= \\
& \sum_{j_{s}=1}^{3} \sum_{\hat{k}=1}^{M} \mathcal{A}_{i_{s}, j_{s}, \hat{j}, \hat{k}}(\widehat{\mathcal{Y}}(t)) \widehat{Y}_{j_{s}, \hat{k}}(t)-\sum_{\hat{k}=1}^{\widehat{M}} \mathcal{M}_{\hat{j}, \hat{k}} \widehat{U}_{\hat{k}}(t),
\end{aligned}
$$

where $\mathcal{A}_{i_{s}, j_{s}, \hat{j}, \hat{k}}(\hat{\mathcal{Y}}(t))$ represents the right-hand-side matrices including nonlinear terms and $\widehat{\mathcal{Y}}(t)$ represents the combined states and nodes array. A similar backward ODE is written for the co-state $\widehat{\xi}_{i_{s} \hat{k}}(t)$ while $\mathcal{B}_{i_{s}, j_{s}, \hat{j}, \hat{k}}(\widehat{\mathcal{Y}}(t))$ represents the nonlinear coefficients in (22), i.e.,

$$
\begin{gathered}
\sum_{\hat{k}=1}^{\widehat{M}} \mathcal{M}_{\hat{j}, \hat{k}} \widehat{\xi}_{i_{s}, \hat{k}}^{\prime}(t)=+\sum_{\hat{k}=1}^{\widehat{M}} \mathcal{M}_{\hat{j}, \hat{k}} R \widehat{Y}_{i_{s} \hat{k}}(t) \\
\quad-\sum_{j_{s}=1}^{3} \sum_{\hat{k}=1}^{\widehat{M}} \mathcal{B}_{i_{s}, j_{s}, \hat{j}, \hat{k}}(\widehat{\mathcal{Y}}(t)) \widehat{\xi}_{j_{s}, \hat{k}}(t) .
\end{gathered}
$$

The essential setup for a general Crank-Nicolson method is to use the midpoint approximation on the integral form of the differential equations followed by an average approximation of the midpoint values, producing from the state ODE for $\widehat{Y}_{i_{s}, \hat{k}, \ell+1}$ at time $t_{\ell}=(\ell-1) * \Delta t$ with $\ell=1: N_{t}$,

$$
\begin{gathered}
\sum_{\hat{k}=1}^{\widehat{M}} \mathcal{M}_{\hat{j}, \hat{k}}\left(\widehat{Y}_{i_{s}, \hat{k}, \ell+1}-\widehat{Y}_{i_{s}, \hat{k}, \ell}\right)=-\Delta t \sum_{\hat{k}=1}^{\widehat{M}} \mathcal{M}_{\hat{j}, \hat{k}} \widehat{U}_{\hat{k}, \ell+0.5} \\
+\Delta t \sum_{j_{s}=1}^{3} \sum_{\hat{k}=1}^{\widehat{M}} \mathcal{A}_{i_{s}, j_{s}, \hat{j}, \hat{k}, \ell+0.5} \widehat{Y}_{j_{s}, \hat{k}, \ell+0.5}
\end{gathered}
$$

where the average approximation at the midpoint is $\widehat{Y}_{j_{s}, \hat{k}, \ell+0.5} \simeq 0.5 *\left(\widehat{Y}_{i_{s}, \hat{k}, \ell+1}+\widehat{Y}_{i_{s}, \hat{k}, \ell}\right)$ and $\widehat{U}_{\hat{k}, \ell+0.5} \simeq 0.5 *$ $\left(\widehat{U}_{\hat{k}, \ell+1}+\widehat{U}_{\hat{k}, \ell}\right)$, compatible with the midpoint approximation in accuracy. The approximation $\mathcal{A}_{i_{s}, j_{s}, \hat{j}, \hat{k}, \ell+0.5}$ is similarly computed. Also, except for backward integration, the co-state $\widehat{\xi}_{i_{s}, \hat{k}, \ell-1}$ satisfies

$$
\begin{gathered}
\sum_{\hat{k}=1}^{\widehat{M}} \mathcal{M}_{\hat{j}, \hat{k}}\left(\widehat{\xi}_{i_{s}, \hat{k}, \ell-1}-\widehat{\xi}_{i_{s}, \hat{k}, \ell}\right)=-\Delta t \sum_{\hat{k}=1}^{\widehat{M}} \mathcal{M}_{\hat{j}, \hat{k}} R \widehat{Y}_{\hat{k}, \ell-0.5} \\
+\Delta t \sum_{j_{s}=1}^{3} \sum_{\hat{k}=1}^{\widehat{M}} \mathcal{B}_{i_{s}, j_{s}, \hat{j}, \hat{k}, \ell-0.5} \widehat{\xi}_{j_{s}, \hat{k}, \ell-0.5},
\end{gathered}
$$

with average approximation at the midpoint, $\widehat{\xi}_{j_{s}, \hat{k}, \ell-0.5} \simeq 0.5 *$ $\left(\widehat{\xi}_{i_{s}, \hat{k}, \ell-1}+\hat{\xi}_{i_{s}, \hat{k}, \ell}\right)$.

Next the predictor-corrector procedure is used to handle the remaining implicit and nonlinear terms. The zeroth corrector, given the final correction $\widehat{Y}_{i_{s}, \hat{j}, \ell+1}$ at time stage $\ell+1$ is the predictor, $\mathrm{YC}_{i_{s}, \hat{j}, \ell+1}^{(0)}=\widehat{Y}_{i_{s}, \hat{j}, \ell}$. This initialization permits finding the $(\gamma+1)$ th correction $\mathrm{YC}_{\hat{j}, \ell+1}^{(\gamma+1)}$ from

$$
\begin{gathered}
\sum_{\hat{k}=1}^{\widehat{M}} \mathcal{M}_{\hat{j}, \hat{k}}\left(\mathrm{YC}_{i_{s}, \hat{k}, \ell+1}^{(\gamma+1)}-\mathrm{YC}_{i_{s}, \hat{k}, \ell}^{(\gamma)}\right)=-\Delta t \sum_{\hat{k}=1}^{\widehat{M}} \mathcal{M}_{\hat{j}, \hat{k}} \\
\cdot \mathrm{UC}_{\hat{k}, \ell+0.5}^{(\gamma)}+\Delta t \sum_{j_{s}=1}^{3} \sum_{\hat{k}=1}^{\widehat{M}} \mathrm{AC}_{i_{s}, j_{s}, \hat{j}, \hat{k}, \ell+0.5}^{(\gamma)} \mathrm{YC}_{j_{s}, \hat{k}, \ell+0.5}^{(\gamma)},
\end{gathered}
$$

where $Y C_{j_{s}, \hat{k}, \ell+0.5}^{(\gamma)}$ and other midpoint terms are evaluated as before by averaging. The final correction at the final time of the state-shot forward iteration, $\widehat{Y}_{\hat{j}, N_{t}+1}$ yields the starting or final-time condition for $\widehat{\xi}_{i_{s}, \hat{k}, N_{t}+1}$ using (14). Then the final correction at the initial time of the costateshot backward iteration produces the initial control condition (23) when $i_{s}=3$ which is used to begin another double for $\delta=2: L$. The algorithm is implemented in MATLAB ${ }^{\mathrm{TM}}$ on a desktop computer.

The data for the numerical parameters are drawn from various sources including Wang et al. [14], [15], Swanson [13] and Murray [11], while unavailable parameters were estimated. The diffusion diagonal vector is $D=[4.2 \mathrm{e}-3,1 . \mathrm{e}-$ $15,0.216] \mathrm{cm}^{2}$ per day. The quadratic cost coefficients are $r_{1}=0.1=q_{1}=q_{3}$ and $s_{3}=0.2$. The net growth coefficient is $a=[1.2 \mathrm{e}-2,8.64 \mathrm{e}-7,11.3]$ per day. The other coefficients are $k_{i}=1, i=1: 2, \alpha_{1,2}=\alpha_{2,1}=\kappa_{2,3}=1 . e-4$ and $\kappa_{1,3}=0.5$. The initial tumor spread and drug concentration are assumed to be Gaussian with scale and state dependent means, spread and weights. Maple ${ }^{\mathrm{TM}} 9.5$ was used to exactly evaluate the integrals of the element matrices off-line.

A sample history of the optimal relative tumor density $Y_{1}^{*}(r, \theta, \psi, t)$ for $r$ over $[0,5]$ in centimeters at fixed $(\theta, \psi)=$ $(\pi, \pi / 2)$ in radians and at quartiles in time of a 5 day 
treatment schedule is given in Figure 2. The initial tumor peak is at $(r, \theta, \psi)=(2.5, \pi, \pi / 2)$. This test case shows significant reduction of the tumor density over the treatment schedule. The computations took 7 hours on a Mac G5 dual

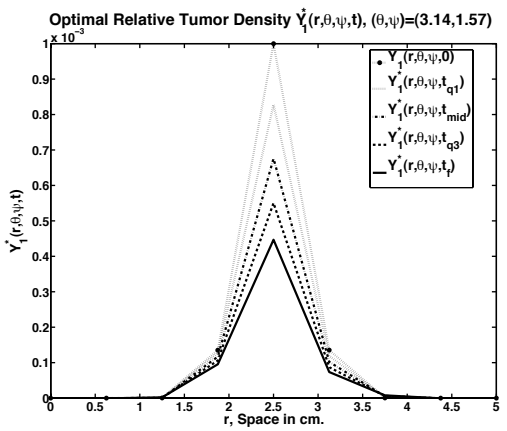

Fig. 2. The optimal, relative tumor density $Y_{1}^{*}(r, \theta, \psi, t)$ versus $r$ at time, $t=0$ days, as a cross-section at $(\theta, \psi)=(\pi, \pi / 2)$ radians, with the initial tumor density peak location with $(r, \theta, \psi)=(2.5, \pi, \pi / 2)$. The grid size is $\left(M_{r}, M_{\theta}, M_{\psi}\right)=(8,8,4)$.

$2 \mathrm{GHz}$, using 2 double shots and a maximum of 2 corrections per shot. Nevertheless, the grid is somewhat coarse due to the high computational complexity of the numerical problem, so more high performance computing [8] would be needed for greater grid refinement.

A more detailed presentation of the initial and final tumor densities are presented for $t=t_{0}=0$ and $t=t_{f}=5$ days is given in Figs. 3(a)-3(b) over the larger $(r, \theta)$ plane section with fixed $\psi=\pi / 2$ showing that the final peak value is small and the tumor has not spread significantly through the rest of the plane in spherical coordinates, although somewhat in the $\theta$-direction but not much in the $r$-direction. The final peak to peak tumor density reduction is $55.3 \%$.

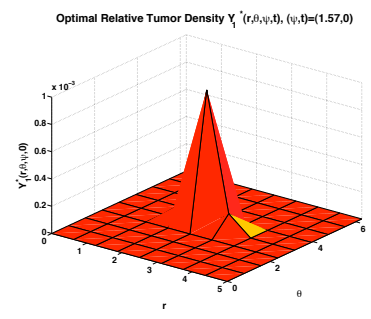

(a) Initial state at $t=0$.

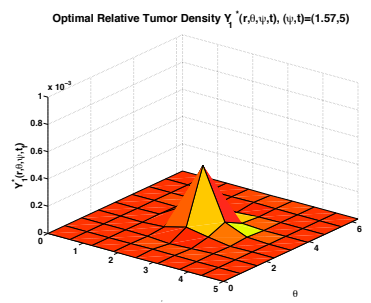

(b) Final state at $t=5$.
Fig. 3. The initial and final relative tumor densities $Y_{1}^{*}(r, \theta, \psi, t)$ when $t=0$ and $t=5$ days, respectively, over $(r, \theta)$ plane $\psi=\pi / 2$ The FEM grid size is $\left(M_{r}, M_{\theta}, M_{\psi}\right)=(8,8,4)$.

\section{Conclusion And Future Directions}

The theory of Galerkin finite elements is used to develop approximations to the distributed parameter optimal control problem of cancer drug delivery to the brain governed by a coupled set of three reaction diffusion PDEs in three space dimensions. The three state variables are the tumor cell density, normal cell density and cancer drug concentration. While the tumor and normal cells are highly coupled through competitive interactions, the concentration is directly controlled by the drug delivery control rate. The optimally con- trolled distributed parameter system is derived by a straightforward calculus of variations technique without resort to an extremely abstract formulation, and that should be useful in other similar scientific or engineering applications.

The system of optimal PDEs is reduced by Galerkin approximations of the state, co-state and control vectors to a system of six ODEs in time with three fundamental element integral coefficient forms: the mass, the stiffness and nonlinear coefficients. The finite element configuration is given for a spherical geometry that can be used to test the optimal drug delivery computations. This finite element configuration will be more amenable to complex brain structures and threedimensional geometries than the finite difference method and low dimension of our earlier work.

Future directions include application to more realistic brain geometries and inhomogeneities.

\section{ACKNOWLEDGMENTS}

The authors gratefully acknowledge Dr. Herbert H. Engelhard of Neurological Surgery in the Medical Center and Professor William D. O'Neill of Bioengineering at the University of Illinois at Chicago for suggesting the problem. We thank a reviewer who gave a good suggestion about the size of the dose.

\section{REFERENCES}

[1] S. P. Chakrabarty, Optimal Control of Drug Delivery to Brain Tumors Using a Distributed Parameters Deterministic Model, $\mathrm{PhD}$ Thesis in Mathematics, University of Illinois at Chicago, July 2006.

[2] S. P. Chakrabarty and F. B. Hanson,"Optimal control of drug delivery to brain tumors for a distributed parameters model," in Proceedings of the American Control Conference 2005, pp. 973-978, 2005.

[3] S. P. Chakrabarty and F. B. Hanson," Optimal control of drug delivery to brain tumors for a test of PDE driven models using the Galerkin finite element Method", in Proceedings of the Conference in Decision and Control and European Control Conference 2005, pp. 1613-1618, 2005.

[4] H. H. Engelhard, "Brain Tumors and the Blood-Brain Barrier," NeuroOncology, The Essentials, pp. 49-53, Thieme Medical Publishers, Inc., New York 2000.

[5] R. A. Gatenby and E. T. Gawlinski, "A Reaction-Diffusion Model of Cancer Invasion," Cancer Research, vol. 56, pp. 5745-5753, December $15,1996$.

[6] M. D. Gunzburger, Perspectives in Flow Control and Optimization, SIAM Series in Design and Control, Philadelphia, 2003.

[7] R. Haberman, Elementary Applied Partial Differential Equations with Fourier Series and Boundary Value Problems, Prentice-Hall, Englewood Cliffs, NJ, 1983.

[8] F. B. Hanson, "Techniques in Computational Stochastic Dynamic Programming," Control and Dynamic Systems: Advances in Theory and Applications, vol. 76, pp. 103-162, Academic Press, New York, April 1996.

[9] J. Mansuri, The Modelling of Tumor Growth Using Reaction-Diffusion Equations, MSc Thesis, Oxford University, 2002.

[10] J. D. Murray, Mathematical Biology I: An Introduction, Springer, New York, 2002.

[11] J. D. Murray, Mathematical Biology II: Spatial Models and Biomedical Applications, Springer, New York, 2003.

[12] G. Sewell, The Numerical Solution of Ordinary and Partial Differential Equations, Academic Press, New York, 1988.

[13] K. R. Swanson, Mathematical Modeling of the Growth and Control of Tumors, PhD Thesis, University of Washington, 1999.

[14] C-H. Wang and J. Li, "Three dimensional simulation of IgG delivery to tumors," Chemical Engineering Science, vol. 53, pp. 3579-3600, 1998.

[15] C-H. Wang, J. Li, C. S. Teo and T. Lee, "The delivery of BCNU to brain tumors," Journal of Controlled Release, vol. 61, pp. 21-41, 1999. 